latory arrest is avoided. The described new perfusion technique with a novel cannula for distal perfusion allows aortic arch procedures at mild hypothermia with preservation of distal organ function.

Initial experience with this technique demonstrated simple handling of the cannula, safety of the perfusion technique, and promising clinical results, with no significant impairment of spinal chord, renal, or intestinal organ function in the first 10 patients. Based on these experiences, we started to use this perfusion technique with $30^{\circ} \mathrm{C}$ mild systemic hypothermia together with ACP as our standard for aortic arch operations. In avoiding ischemia and deep hypothermia, this technique might help to reduce CPB time and deep hypothermia-related side effects.

\section{References}

1. Sabik JF, Lytle BW, McCarthy PM, Cosgrove DM. Axillary artery: an alternative site of arterial cannulation for patients with extensive aortic and peripheral vascular disease. J Thorac Cardiovasc Surg. 1995;109:885-90.

2. Takagi H, Mori Y, Iwata H, Umeda Y, Matsuno Y, Hirose H. Aortic balloon occlusion catheter with perfusion lumen for protection of lower body during distal anastomosis in aortic arch repair. J Thorac Cardiovasc Surg. 2002;123:1006-8.

3. Kodell CT, Hess PJ, Beaver TM, Clark D, Martin TD. Distal aortic perfusion during arch reconstruction: another tool for the aortic surgeon. Ann Thorac Surg. 2004;78:2196-8.

4. Bakhtiary F, Dogan S, Dzemali O, Kleine P, Moritz A, Aybek T. Mild hypothermia $\left(30^{\circ} \mathrm{C}\right)$ and antegrade cerebral perfusion in aortic arch operations. J Thorac Cardiovasc Surg. 2006;132:153-4.

\title{
Alternative approach for stent grafting of the thoracic descending aorta: The antegrade right axillary approach
}

\author{
Bertrand Marcheix, MD, MSc, ${ }^{\text {a }}$ Philippe Demers, MD, MSc, ${ }^{a}$ \\ Frédéric Vanden Eynden, MD, ${ }^{a}$ Yoan Lamarche, MD, MSc, ${ }^{a}$ Éric Therasse, MD, ${ }^{b}$ \\ and Raymond Cartier, MD, ${ }^{a}$ Montreal, Quebec, Canada
}

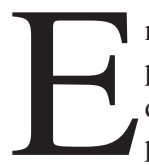
ndovascular procedures are new options for high-risk patients presenting with a pathologic condition of the descending thoracic aorta. We report the case of a patient who underwent stent grafting of the descending thoracic aorta to treat a symptomatic penetrating ulcer. Because a conventional femoral approach was impossible, an antegrade right axillary approach was used.

\section{Clinical Summary}

A 53-year-old man was referred to us with a progressing intramural hematoma associated with a penetrating aortic ulcer of the descending thoracic aorta (T7-T8 level; Figure 1, A-E). Despite optimal medical therapy, the patient remained symptomatic, and 3 consecutive computed tomographic (CT) scans performed at 1-week intervals confirmed the progression of the ulcer. Because of the patient's chronic obstructive pulmonary disease, left ven-

From the Departments of Cardiovascular Surgery ${ }^{\mathrm{a}}$ and Interventional Radiology, ${ }^{\mathrm{b}}$ Montreal Heart Institute, and the Université de Montréal, Montreal, Quebec, Canada.

Received for publication Oct 25, 2006; revisions received Dec 15, 2006; accepted for publication Jan 5, 2007.

Address for reprints: Raymond Cartier, MD, Department of Cardiovascular Surgery, Montreal Heart Institute, 5000 Belanger St, Montreal, Quebec H1T 1C8, Canada (E-mail: rc2910@aol.com).

J Thorac Cardiovasc Surg 2007;133:1639-41

$0022-5223 / \$ 32.00$

Copyright $\odot 2007$ by The American Association for Thoracic Surgery doi:10.1016/j.jtcvs.2007.01.031 tricular dysfunction, and chronic occlusion of the infrarenal aorta (Figure 1, F), conventional open-chest surgery was judged to be prohibitive. Two different stent grafting strategies were considered. The first option

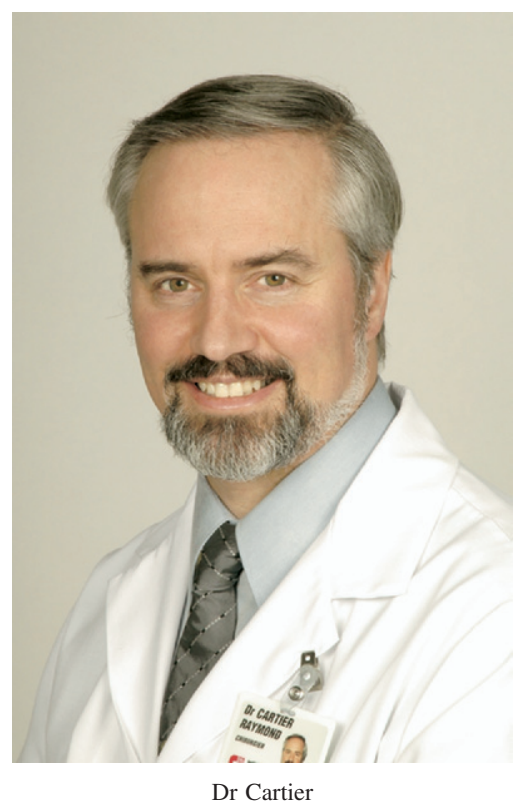
was to proceed to a classic retrograde stent graft delivery after an aortobi-iliac bypass, and the second was to deliver the stent graft in an antegrade fashion through the right axillary artery approach. The patient did not complain of significant claudication and refused conventional abdominal surgery. Axillary and subclavian arteries were patent, with diameters ranging between 8.5 and $9 \mathrm{~mm}$ (Figure $1, G$ ). The CT scan revealed no significant atheromatous lesions on the aortic arch, and hence the second option was selected. The procedure was performed after the patient underwent general anesthesia. Digital subtraction angiography and transesophageal echocardiographic guidance were used. Before stent positioning, an aortography was performed through the left brachial artery with a pigtail catheter (Cordis; Johnson \& Johnson, Warren, NJ). After intravenous administration of 5000 IU of heparin, the right axillary artery was dissected out, and a 10-mm Dacron conduit was sutured in a terminolateral fashion. The $22 \mathrm{~F}$ delivery system (Valiant Medtronic, Minneapolis, Minn) was introduced by the axillary conduit on a 260-cm-long superstiff guide wire (Back-Up Meier; Boston Scientific, Oakland, NJ; Figure 1, $H$ 

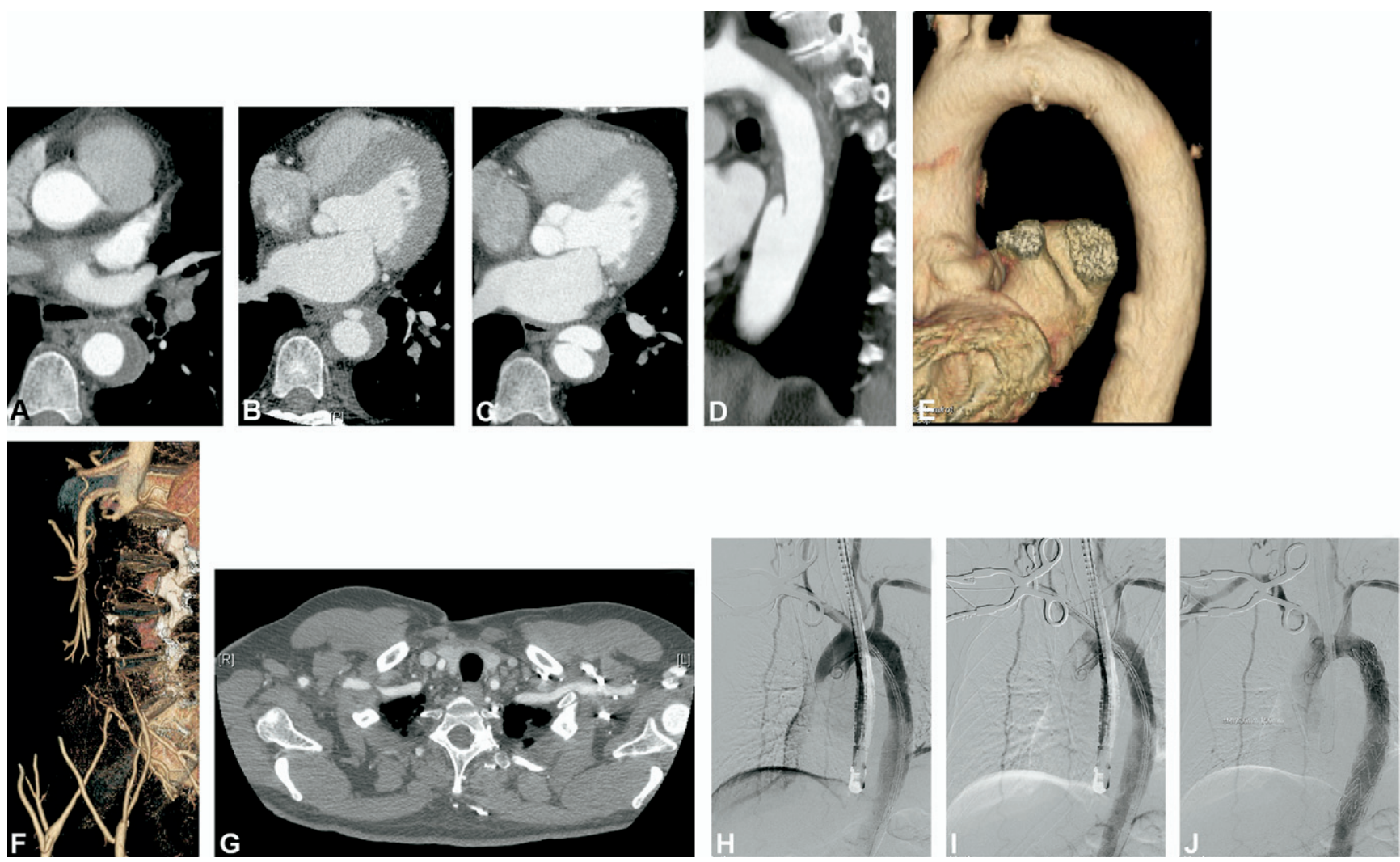

Figure 1. Computed tomographic scan mediastinal settings and injection of contrast demonstrating an aortic intramural hematoma complicated by an aortic ulcer. A-C, Axial views; D, sagittal reconstruction; E, 3-dimensional reconstruction showing the location of the aortic ulcer; $F$, occlusion of the abdominal infrarenal aorta; $G$, axial view of the axillary and subclavian arteries; $\mathrm{H}$ and $\mathrm{I}$, intraoperative angiography views showing the progression of the delivery device through the right axillary artery; J, control angiography confirming patency of the stent graft and the absence of endoleak.

and $I$ ). A Valiant prosthesis (Proximal Free Flow straight, $129 \times$ $30 \mathrm{~mm}$ ) was positioned at the level of the aortic ulcer and deployed under controlled hypotension. Control angiography (Figure 1, J) and transesophageal echocardiography demonstrated adequate coverage of the aortic ulcer and absence of endovascular leak. The proximal end of the Dacron conduit was left in place and simply sutured to achieve hemostasis. Except for a mild left pleural effusion, the postoperative course was uneventful, and the follow-up CT scan at 6 months showed complete thrombosis of the aortic ulcer.

\section{Discussion}

Endovascular management of pathologic conditions of the descending thoracic aorta has been reported to be a safe and efficient alternative to conventional open-chest surgery in high-risk patients. ${ }^{1}$ Our patient presented with the unusual combination of thoracic aortic intramural hematoma with a penetrating ulcer and occlusive disease of the infrarenal abdominal aorta, precluding the classical retrograde femoral approach for stent graft deployment. Despite optimal medical therapy, the patient remained symptomatic and the ulcer progressed, making the surgical option mandatory. ${ }^{2,3}$ Concomitant treatment of the abdominal aorta and the thoracic aortic ulcer was the primary surgical strategy of choice. Although less invasive, endovascular procedures still carry the risk of immediate or delayed paraplegia, especially when the T9-T12 aortic segment is covered by the stent graft or when the procedure is performed concomitantly with surgical correction of infrarenal aortic aneurysms. In the Stanford series $(n=103)$, paraplegia was reported in 2 patients having undergone simultaneous repair of abdominal and thoracic aneurysms and in a third patient having undergone prior aortic aneurysm repair years before. ${ }^{4,5}$ For these reasons, we considered combining infrarenal aortic surgery with stent grafting of the thoracic aorta, a situation that increases the risk of paraplegia.

On angiographic and Doppler investigation, both axillary arteries were patent, with diameters of greater than $8 \mathrm{~mm}$, and no significant arch disease was documented, demonstrating the axillary approach to be a safe one.

The left axillary artery could have been a better choice than the right one by preventing the manipulation of the device close to the origin of the supra-aortic vessels, with potential risk for cerebral embolisms. In our patient the bilateral pigtails initially inserted through both brachial approaches showed the right axillary approach to be straighter. Direct cannulation of the artery did not 
seem to be a reasonable strategy because the arterial wall looked thin and fragile. The use of an end-to-side 10-mm Dacron conduit was believed to be a safer approach.

In conclusion, in cases in which the femoral approach is not suitable, the antegrade right axillary approach constitutes a safe and efficient alternative for stent grafting of the thoracic aorta, as long as the arch and axillary anatomy are favorable.

\section{References}

1. Dake MD, Miller DC, Semba CP, Mitchell RS, Walker PJ, Liddell RP. Transluminal placement of endovascular stent/grafts for the treatment of descending thoracic aortic aneurysms. N Engl J Med. 1994;331:1729-34.
2. Tittle SL, Lynch RJ, Cole PE, Singh HS, Rizzo JA, Kopf GS, et al. Midterm follow-up of penetrating ulcer and intramural hematoma of the aorta. J Thorac Cardiovasc Surg. 2002;123:1051-9.

3. Demers P, Miller DC, Mitchell RS, Kee ST, Chagonjian L, Dake MD. Stent-graft repair of penetrating atherosclerotic ulcers in the descending thoracic aorta: mid-term results. Ann Thorac Surg. 2004; 77:81-6.

4. Moon MR, Mitchell RS, Dake MD, Zarins CK, Fann JI, Miller DC. Simultaneous abdominal aortic replacement and thoracic stent-graft placement for multilevel aortic disease. J Vasc Surg. 1997;25:33240.

5. Dake MD, Miller DC, Mitchell RS, Semba CP, Moore KA, Sakai T. The "first generation" of endovascular stent-grafts for patients with aneurysms of the descending thoracic aorta. J Thorac Cardiovasc Surg. 1998;116:689-704

\title{
Surgical treatment of tracheoinnominate fistula after stent-graft implantation
}

\author{
Enrico Maria Marone, MD, ${ }^{a}$ Gloria Esposito, MD, ${ }^{a}$ Andrea Kahlberg, MD, ${ }^{a}$ \\ Yamume Tshomba, MD, ${ }^{a}$ Chiara Brioschi, MD, ${ }^{a}$ Piero Zannini, MD, ${ }^{\mathrm{b}}$ and \\ Roberto Chiesa, MD, ${ }^{a}$ Milan, Italy
}

$\mathrm{P}$

rotrusion of stent-grafts into adjacent structures is an uncommon but life-threatening complication of endovascular procedures and may result in fistulization. Tracheoinnominate fistulization is a devastating and often fatal condition, requiring prompt diagnosis and appropriate surgical treatment. ${ }^{1}$ This report describes a case of a tracheoinnominate fistula as a delayed complication of a posttraumatic pseudoaneurysm endovascular repair.

\section{Clinical Summary}

A 21-year-old Asian man with a 5-cm posttraumatic pseudoaneurysm of the innominate artery was treated at the referring hospital by implantation of three covered stents from the origin of the innominate artery to the proximal tract of the right common carotid artery. Postoperative computed tomographic scans showed complete pseudoaneurysm exclusion and right subclavian artery occlusion at the origin, reperfused by the right vertebral artery. The

From the Chairs of Vascular Surgery and Thoracic Surgery, ${ }^{\mathrm{b}}$ Department of Thoracic and Cardiovascular Surgery, Scientific Institute H. San Raffaele, Vita-Salute University School of Medicine, Milan, Italy,

Received for publication Dec 6, 2006; accepted for publication Dec 12, 2006.

Address for reprints: Enrico Maria Marone, MD, Chair of Vascular Surgery, Scientific Institute H. San Raffaele, Vita-Salute University School of Medicine, Via Olgettina 60, 20132, Milan, Italy (E-mail: marone. enrico@hsr.it)

J Thorac Cardiovasc Surg 2007;133:1641-3

$0022-5223 / \$ 32.00$

Copyright $\odot 2007$ by The American Association for Thoracic Surgery doi:10.1016/j.jtcvs.2006.12.063 postoperative course was complicated by a tracheal ring laceration

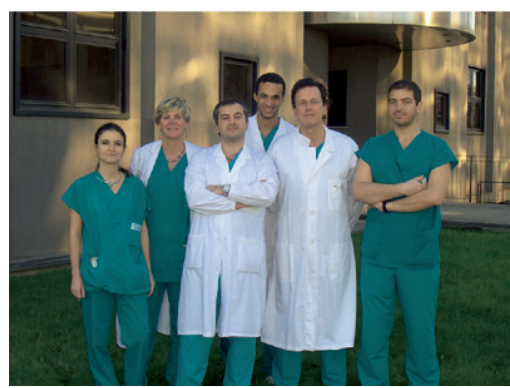

Drs Brioschi, Esposito, Marone, Tshomba, Prof Chiesa, and Dr Kahlberg (left to right) (requiring endoscopic toilet and tracheostomy), pulmonary infection, and sepsis. The patient was given antibiotic therapy and discharged in stable conditions.

Two months later, a scheduled fiberbronchoscopy, followed by magnetic resonance imaging of the chest, showed erosion of stentgrafts into the tracheal lumen for a $3-\mathrm{cm}$ long segment, with multiple ulcerations of the right lateral wall. The patient was transferred to our institution for further treatment. On the first day, he had several episodes of hemoptysis. Preoperative imaging confirmed protrusion of the stent-grafts into the tracheal lumen (Figure 1). The patient underwent right cervicosternotomy and surgical exploration of the cervicothoracic region. A fistula was found between the innominate artery, where the stent-grafts had been placed, and the trachea. The grafts seemed also to be excessively oversized relative to the artery diameter. We proceeded to excision of the innominate artery and stent-grafts, extra-anatomic cerebral revascularization (through a left-to-right common carotid artery crossover bypass with a 6-mm expanded polytetrafluoroethylene ringed graft), and tracheal reconstruction with an autologous patch of thymus, pericardium, and arterial wall.

Despite initial hemodynamic stability, hemothorax, hemopericardium, and massive hemoptysis developed in the postoperative period as a result of active bleeding from the innominate artery stump. Consequently, the patient underwent hypothermic cardiac arrest and urgent resternotomy. The aortic arch wall was found to be lacerated and was sutured with a bovine pericardial patch (Figure 2). Dehiscence of tracheal reconstruction was treated by sealing a pedicled left internal thoracic artery, parietal pleura, and 